SINP/TNP/06-08

hep-ph/0604135

\title{
PDF and scale uncertainties of various DY distributions in ADD and RS models at hadron colliders
}

\author{
M. C. $\operatorname{Kumar}^{a, b} *$, Prakash Mathews ${ }^{a} \dagger$, V. Ravindran ${ }^{c} \ddagger$ \\ a) Saha Institute of Nuclear Physics, 1/AF Bidhan Nagar, Kolkata 700 064, India. \\ b) School of Physics, University of Hyderabad, Hyderabad 500 046, India. \\ c) Harish-Chandra Research Institute, Chhatnag Road, Jhunsi, Allahabad, India.
}

\begin{abstract}
In the extra dimension models of ADD and RS we study the dependence of the various parton distribution functions on observable of Drell-Yan process to NLO in QCD at LHC and Tevatron energies. Uncertainties at LHC due to factorisation scales in going from leading to next-to-leading order in QCD for the various distributions get reduced by about 2.75 times for a $\mu_{F}$ range $0.5 Q<\mu_{F}<1.5 Q$. Further uncertainties arising from the error on experimental data are estimated using the MRST parton distribution functions.
\end{abstract}

*mc.kumar@saha.ac.in

†prakash.mathews@saha.ac.in

${ }^{\ddagger}$ ravindra@mri.ernet.in 


\section{Introduction}

The gauge hierarchy problem has been one of the main motivations for physics beyond the Standard Model (SM). One of the directions that has emerged, looks at why gravity appears weak as compared to the other three interactions of the SM. This apparent weakness has been accounted for by the existence of either large extra spatial dimensions ADD model [1] or warped extra dimension RS model [2]. In either case the fundamental Planck scale could be of the order of a $\mathrm{TeV}$ and hence a possible explanation of the hierarchy. In both these models only gravity is allowed to propagate the extra dimensions while the SM particles are constrained on a 3-brane. This consequently leads to Kaluza-Klein (KK) modes in 4-dimensions, which in ADD and RS models have distinct KK spectrum and their effective interaction with the SM model particles. The experimental signatures of these KK modes have been of intense phenomenological activity. With the closure of LEP and the advent of LHC the focus now shifts to hadron colliders.

At hadron colliders, it is important to have a precise knowledge of the parton distributions functions (PDFs) to predict production cross sections of both signals and backgrounds. These universal PDFs are non-perturbative inputs that are extracted from global fits to available data on deep-inelastic scattering (DIS), Drell-Yan (DY) and other hadronic processes. They describe the momentum distribution of the partons in a proton and various groups have parametrised the PDFs for a wide range of proton

momentum fraction $x$ carried by the parton and for the center-of-mass energy $Q^{2}$ at which the process takes place. Parametrisation of PDFs to a particular order in QCD would involve various theoretical and experimental uncertainties. Recently there has been a series of papers [3]-[ $]$ which for the first time have calculated the next-toleading order (NLO) QCD corrections to various distributions of DY process for both ADD and RS model. In [4, NLO-QCD corrections to dilepton production at hadron colliders in the ADD model were presented for the first time, this was extended to 
the RS model in [5]. Further in [6] we have considered the double differential cross section, $d^{2} \sigma / d Q^{2} / d \cos \theta^{*}$, for dilepton production in models with large and warped extra dimensions. The $\cos \theta^{*}$ distribution is the one that is actually used by experiments and hence is of particular importance. These NLO results would certainly reduce one aspect of the theoretical uncertainties as results prior to this calculation were only at leading order (LO) in QCD for process involving gravity.

In [4, 5, 6] we have quantified the theoretical uncertainties coming from the QCD corrections by computing all the processes that enter at NLO level. Unlike the standard model contribution to DY, the extra-dimensional models bring in more processes even at the LO level. For example, in these models, the gluon initiated process enter at the LO level in addition to quark anti-quark initiated process. At LHC, the gluon initiated process is more sensitive to factorisation scale compared to quark initiated process that necessitated the relevance of NLO computation. It was found in [4, 5, 6] that the NLO corrections are considerably large and the factorisation scale uncertainty goes down significantly with these corrections as expected. Our entire analysis is model independent because the QCD corrections factor out from the model dependent quantities. In [4, 5, 6], we used the MRST parton density sets. It is well known that the different PDF sets themselves can affect the theoretical predictions and it is important to quantify these effects in the observable that could probe new physics. With this in mind we have performed a model independent analysis on uncertainties coming from the choice of PDF sets in order to make our predictions more reliable. In this paper we have looked at the dependence due to various PDFs for the production of dilepton at LHC and Tevatron including gravity effects in the ADD and RS models incorporating the NLO QCD corrections. The PDF sets used in this study are Alekhin [7, CTEQ [8] and MRST [9]. Dependence on PDF sets is also compared with experimental errors that enter the parametrisation of the PDF, which are now available to NLO QCD [10, 11]. For this purpose we used the MRST distribution [11] as a typical case. The 
dependence of factorisation scale $\mu_{F}$ and renormalisation scale $\mu_{R}$ in going from LO to NLO is also studied.

For the ADD model we also study the dependence on UV cutoff of the KK mode sum by keeping the UV cutoff different from the scale of the model $M_{S}$. This prescription allows us to study the cutoff dependence. The dependence of the cross section on the number of extra dimension as a result of this prescription is similar to that of the real graviton production case. The ADD model is a low energy effective theory valid below the scale $M_{S}$, it is conventional to equate the cutoff to the scale of the effective theory [12, 13].

The plan of the paper is as follows. In section 2 we describe the extra dimension models studied and describe the parameters of the models. In section 3 we discuss the theoretical uncertainties viz. the PDF uncertainties, renormalisation and factorisation scale dependences. The improvement of the scale dependence in going from LO to NLO is also discussed. In section 4 we look at the dependence due to the error on the data and estimate the experimental error for a few observable using the MRST PDF. Finally we summarise our results in section 5 .

\section{Extra Dimension Models}

Extra dimension models that allow gravity to propagate the extra dimensions would in 4-dimensions have KK modes which couple to SM particles through the energy momentum tensor. The Feynman rules of the KK mode interactions with the SM fields are given in [12, 13. Due to the different methods of compactification of the extra dimensions in ADD and RS models, their KK spectrum are very distinct. Experimental signature of extra dimensions would correspond to deviation from SM predictions due to the virtual exchange of KK modes or direct production of KK modes at a collider.

In the $\mathrm{ADD}$ case, there is a tower of KK modes which are almost degenerate in energy and a sum over these KK modes gives an observable effect. In the case of 
dilepon production, in addition to the SM photon and $Z$ production modes, one has to take into account virtual KK modes. Performing the sum over the virtual KK modes leads to an integral which has to be regulated by an UV cutoff. The propagator after the KK mode summation becomes

$$
\kappa^{2} \mathcal{D}\left(Q^{2}\right) \equiv \kappa^{2} \sum_{n} \frac{1}{Q^{2}-m_{n}^{2}+i \epsilon}=\frac{16 \pi}{M_{S}^{4}}\left(\frac{Q}{M_{S}}\right)^{d-2} I\left(\frac{\Lambda_{c}}{Q}\right),
$$

where $\kappa=\sqrt{16 \pi} / M_{P}$ is the strength of the gravitational coupling to the SM particles, $m_{n}$ the mass of KK modes, $d$ is the number of extra dimensions and $M_{S}$ is the scale of the $4+d$ dimensional theory. The summation over the non-resonant KK modes yields $I\left(\Lambda_{c} / Q\right)$ [13. Conventionally the UV cutoff $\Lambda_{c}$ is identified with the scale of the extra dimension theory $M_{S}$, which simplifies the expression giving a mild dependence on the number of extra dimensions [12, 13].

In this analysis, we have kept the cutoff $\Lambda_{c}$ different from $M_{S} \S$. Note that the summation of KK modes in Eq. (11), modifies the $M_{P}$ suppression to $M_{S}$ suppression. The ADD model is a effective low energy theory valid below the scale $M_{S}$, for consistency it is essential to satisfy the condition $Q<\Lambda_{c}<M_{S}$. The parameters of the ADD model are $M_{S}$ the scale of the $4+d$ dimensional theory and $d$ the number of extra spatial dimensions. If $\Lambda_{c} \neq M_{S}$ then there is an additional parameter. We have studied the dependence of the cross section on the cutoff $\Lambda_{c}=\alpha M_{S}$ and varied $\alpha=0.7-1$. In Fig. 1a we see that the cross section decreases as we lower the cutoff $\Lambda_{c}$. The corresponding K-factor also decreases for lower cutoff Fig. 11b. Dependence of the cross section on the number of extra dimensions $d$ is shown in Fig. 11 for $\Lambda_{c}=M_{S}$, the cross section decreases as $d$ increases. Reducing $\Lambda_{c}$ decreases the cross sections and if $d$ is increased it brings down the cross section much faster Fig. 1 $\mathrm{d}$. Large extra dimension searches in the dimuon channel at the Tevatron [15] have put bounds on $M_{S}$ in the range $0.8-1.27 \mathrm{TeV}$. For the analysis, they have used the double differential

\footnotetext{
${ }^{\S}$ Effects of the various UV cutoff methods on the low scale quantum gravity model have been discussed in 14 .
} 
cross section with respect to invariant mass and the $\cos \theta^{*}$ [16].

In the RS model, the gravity propagate one extra dimension which is warped by an exponential factor $\exp (-\pi k L)$, where $L$ is the compactification length and $k$ is the curvature of the $A d S_{5}$ space-time. The parameters of the RS model are $m_{0}=$ $k \exp (-\pi k L)$ which sets the mass scale of the KK modes and $c_{0}=k / M_{P}$ the effective coupling. The higher KK modes have enhanced coupling to SM particles due to the warp factor and decouple from the zero mode, which is as usual $M_{P}$ suppressed. RS KK spectrum is distinct from the ADD case and hence the summation of the KK modes that contribute to the virtual process would also be different. The function $\mathcal{D}\left(Q^{2}\right)$ in the $\mathrm{KK}$ mode propagator results from summing over the resonant $\mathrm{KK}$ modes and is given by

$$
\mathcal{D}\left(Q^{2}\right)=\sum_{n=1}^{\infty} \frac{1}{Q^{2}-M_{n}^{2}+i M_{n} \Gamma_{n}} \equiv \frac{\lambda}{m_{0}^{2}},
$$

where $M_{n}$ are the masses of the individual resonances and $\Gamma_{n}$ are the corresponding decay widths. The graviton widths are obtained by calculating their decays into final states involving SM particles. $\lambda$ is defined as

$$
\lambda\left(x_{s}\right)=\sum_{n=1}^{\infty} \frac{x_{s}^{2}-x_{n}^{2}-i \frac{\Gamma_{n}}{m_{0}} x_{n}}{x_{s}^{2}-x_{n}^{2}+\frac{\Gamma_{n}}{m_{0}} x_{n}},
$$

where $x_{s}=Q / m_{0}$. We have to sum over all the resonances to get the value of $\lambda\left(x_{s}\right)$, which is done numerically for a given value of $x_{s}$. Searches for the RS KK modes at Tevatron in the dielectron, dimuon and digamma channel [17] have yielded a lower limit between 250 - $785 \mathrm{GeV}$ depending on the coupling to the SM particles.

\section{Theoretical uncertainties}

In the QCD improved parton model the hadronic cross section can be expressed in terms of pertubatively calculable partonic cross sections denoted by $\hat{\sigma}^{a b}\left(\tau, Q^{2}, \mu_{F}\right)$ convoluted with appropriate non perturbative partonic flux $\Phi_{a b}\left(\tau, \mu_{F}\right)$ at a factorisation scale $\mu_{F}$. The subprocess cross section is a perturbative expansion in the strong 
coupling constant $\alpha_{s}\left(\mu_{R}\right)$ and is calculated order by order in $\alpha_{s}$. Here $\mu_{R}$ is the renormalisation scale and $\tau=Q^{2} / S$ is the DY scaling variable. In perturbative $\mathrm{QCD}$, the unknown higher order corrections and the scale uncertainties are strongly correlated. The factorisation of mass singularities from the perturbatively calculable partonic cross sections leads to the introduction of factorisation scale $\mu_{F}$ in both non-perturbative partonic flux $\Phi_{a b}\left(\mu_{F}\right)$ as well as the finite partonic cross sections $d \hat{\sigma}_{a b}\left(x, \mu_{F}\right)$. Even though the choice of the scale is guided by the hard scale of the problem, the exact value does not come from the theory. The PDFs and partonic cross sections satisfy renormalisation group equations such that the hadronic cross section is independent of the factorisation scale $\mu_{F}$. In addition to the factorisation scale, the partonic cross sections are dependent on the renormalisation scale $\mu_{R}$. The choice of the scale is again arbitrary. Even though this is an advantage to choose appropriately to do perturbative calculations, it also introduces theoretical uncertainties through the size of unknown higher order corrections. Usually, one chooses this scale such that the perturbative methods can be applied and then computes higher order corrections sufficiently such that the exact choice of this scale becomes almost immaterial. Gravity couples to the SM fields via its energy momentum tensor, and the calculations are done in the high energy limits where masses of the SM particles are ignored. Only parameter that requires UV renormalisation is the strong coupling constant, because of this we have the following expansion for the mass factorised partonic cross section:

$$
d \hat{\sigma}_{a b}\left(z, \mu_{F}^{2}\right)=\sum_{i=0}^{\infty} a_{s}^{i}\left(\mu_{R}^{2}\right) d \hat{\sigma}_{a b}^{(i)}\left(z, \mu_{F}^{2}, \mu_{R}^{2}\right),
$$

where the coupling constant satisfies standard renormalisation group equation. Since we are only interested in the NLO order corrections, the Altarelli-Parisi kernels $P^{(0)}(z)$, $P^{(1)}(z)$ and the coefficients $\beta_{0}, \beta_{1}$ are sufficient for our analysis. The scale uncertainties come about from the truncation of the perturbative series. Unlike the perturbatively calculable partonic cross sections, the PDFs being non-perturbative in nature are extracted from various experiments. These are fitted at a scale of the experiments and 
then evolved according to the AP evolution equations to any other relevant scale. They are not only sensitive to experimental errors but also to theoretical uncertainties that enter through the partonic cross section calculations and the splitting functions that are known only to certain orders in strong coupling constant in perturbative QCD. Here, we mainly concentrate on the uncertainties coming from PDFs in detail and quantify their impact on the new physics searches in extra dimensional models.

\subsection{PDF uncertainty}

We first focus on the uncertainties coming from different PDF sets. The parton flux factor for both LHC and Tevatron would give an idea as to which component would be dominant in the kinematical region of interest. This flux factor enters the cross section. The gluon flux is clearly much larger in the kinematical region of interest at LHC and for Tevatron the $q \bar{q}$ flux is the dominant contribution.

In the context of extra dimension theories we consider the dilepton production at LHC and Tevatron for both large extra dimension and warped extra dimension models. The process of interest is $P_{1}\left(p_{1}\right)+P_{2}\left(p_{2}\right) \rightarrow \mu^{+}\left(l_{1}\right)+\mu^{-}\left(l_{2}\right)+X\left(P_{X}\right)$, where $P_{1}$ and $P_{2}$ are the incoming hadrons, $\mu^{ \pm}$are the final lepton pair and $X$ the final inclusive hadronic state. The dilepton in these models could also be produced from the exchange of a KK mode in addition to the usual SM gauge boson exchange. Hence at LO itself the $g g$ subprocess could contribute to the dilepton production via a KK mode exchange in addition to the $q \bar{q}$ subprocess.

For both new physics searches and precision SM physics at hadron colliders it is essential to understand the uncertainties associated with PDFs. We essentially study to what extent the cross sections depend on the various PDFs viz. Alekhin [7, CTEQ [8] and MRST [9]. In the Table 1, we have tabulated the particular PDF that is chosen

for the study and also the corresponding $\Lambda_{Q C D}$ parameter that is used to determine 
the strong coupling $\alpha_{s}$.

\begin{tabular}{|l|l||l|l|}
\hline \multicolumn{2}{|c||}{ LO } & \multicolumn{2}{c|}{ NLO } \\
\hline PDF & $\Lambda_{Q C D}(\mathrm{GeV})$ & PDF & $\Lambda_{Q C D}(\mathrm{GeV})$ \\
\hline \hline MRST2001 LO & 0.220 & MRST2001 NLO & 0.323 \\
CTEQ6L & 0.326 & CTEQ6M & 0.326 \\
\hline
\end{tabular}

Table 1: The PDF set used in the analysis along with the respective $\Lambda_{Q C D}$.

These groups perform a global analysis of a wide range of DIS and other scattering data to get best fits to a particular order in QCD. Though all these parametrisation satisfies the general constraints, they could differ from each other. This is expected as PDFs are not by itself physical quantities and are extracted subject to experimental and theoretical uncertainties and various assumptions and initial conditions used by the different groups. Differences among the various PDFs would translate as uncertainties on the physical observable.

To NLO in QCD for various PDFs, we now present the comparison plots for the following differential distributions

$$
\frac{d \sigma}{d Q}, \quad \frac{d^{2} \sigma}{d Q d Y}, \quad \frac{d^{2} \sigma}{d Q d \cos \theta^{*}}
$$

We would look at the invariant mass distribution $Q$, the double differential cross section with respect to $Q$ and rapidity $Y$ and the double differential cross section with respect to $Q$ and $\cos \theta^{*}$. The angle $\theta^{*}$ is the angle between the final state lepton momenta and the initial state hadron momenta in the c.o.m frame of the lepton pair. The corresponding K-factor which is the ratio of NLO to LO of the above distributions are also plotted for the various PDFs. For the double differential cross section we fix the invariant mass $Q$ in the region of interest of extra dimensions and plot the cross section with respect to rapidity $Y$ and $\cos \theta^{*}$. The first two distributions in Eq. (15) are $\cos \theta^{*}$ integrated distributions and hence are independent of the interference between

\footnotetext{
IIn the case of Alekhin the PDF itself generates the value of $\alpha_{s}$ and is hence not tabulated.
} 
the SM background and the low scale gravity effects [4. The double differential with respect to $Q$ and $\cos \theta^{*}$ would contain the interference terms, but numerically it is not very significant [6]. Consequently even for the $\cos \theta^{*}$ distributions we can express the $K$ factor of the model involving both SM and gravity as

$$
K^{(S M+G R)}(Q)=\frac{K^{S M}+K^{G R} K^{(0)}}{1+K^{(0)}},
$$

where $K^{G R}$ is the $K$ factor of the pure gravity part. We have introduced a quantity $K^{(0)}$, defined as the ratio of the LO distribution of gravity to SM, given by

$$
K^{(0)}(Q)=\left[\frac{d \sigma_{L O}^{S M}(Q)}{d Q}\right]^{-1}\left[\frac{d \sigma_{L O}^{G R}(Q)}{d Q}\right]
$$

The behaviour of $K^{(0)}(Q)$ is governed by the competing coupling constants of SM and gravity and the parton fluxes involved. Basically the factor $K^{(0)}$ is an indicator as to the source of the total $K^{(S M+G R)}$-factor. $K^{(0)}(Q)$ as a function of $Q$ rises much faster for LHC than Tevatron and reaches 1 much earlier. Since the $g g$ subprocess contributes at LO itself for the gravity mediated process, the gravity effects are much larger at the LHC where the gluon flux is much larger. This would also result in larger K-factor for the process at LHC at large $\mathrm{Q}$ where the gravity contribution dominates. At Tevatron since the gluon flux is smaller the K-factor is similar to the SM K-factor.

For both ADD and RS models the signal for new physics is the excess of events in the total cross section or the various distribution over the SM background. If we restrict ourself to these extra dimensional models, the signal is due to the effect of the KK modes and can not be mimicked by the SM. We would like to emphasise that we are not analysing the existing Tevatron data to extract bounds on the ADD and RS parameters, which would need a full hadron-level simulation, but estimate the various uncertainties to NLO in QCD by choosing typical representative values for the ADD and RS parameters.

We begin with the ADD model wherein we have chosen $d=3$ and $M_{S}=2 \mathrm{TeV}$. In Fig. 2 a the cross section is plotted as a function of the invariant mass $Q$ of the dilepton 
at LHC for the various PDFs. There is only a mild dependence on the difference in the PDFs, but when plotted for the corresponding K-factor then the PDF dependence is larger for both low and high values of $Q$, Fig. 2 b. At low $Q$ it is the SM part which contributes to the K-factor while at high $Q$ it is the beyond SM effects that contribute. At low $Q$ where the K-factor is due to SM part, MRST and CTEQ are similar, while Alekhin is smaller. At large $Q$ the K-factor is due to the gravity part and here CTEQ is larger.

For the double differential cross section with respect to invariant mass distribution and rapidity $Y$ Fig. 3a, we have plotted as a function of rapidity $Y$ for a fixed $Q=0.7$ TeV. Only in the central rapidity region do the PDFs differ, with MRST being the dominant while CTEQ is the smallest. The K-factor is quite large at the central rapidity region and would range from 1.5 - 1.6 depending on the PDF used. The general behaviour of the K-factor is similar for MRST and Alekhin. At large rapidities $y= \pm 2$ the K-factors are quite different with Alekhin being 1.25 while CTEQ the largest is 1.45. For $Q=0.7 \mathrm{TeV}$ the $\mathrm{K}$ factor is large which we can see from Fig. $2 \mathrm{~b}$, wherein the dominant contribution is from the gravity mediated $g g$ initiated subprocess.

In Fig. 3re we have plotted the double differential cross section with respect to $Q$ and $\cos \theta^{*}$ as a function of $\cos \theta^{*}$ for a fixed $Q=0.7 \mathrm{TeV}$. MRST gives the largest and CTEQ the least with Alekhin being a central value in the spread. The difference exists for the full range of $\cos \theta^{*}$. The SM background has a different $\cos \theta^{*}$ dependence. The interference of the SM and the gravity effect is not zero for the $\cos \theta^{*}$ distribution but does not contribute significantly. The K-factor for central $\cos \theta^{*}=0$ region is about 1.52 but differ with PDFs as $\cos \theta^{*} \rightarrow \pm 1$, Fig. 3d. Since there is no $g g$ initiated process in the SM background to NLO the K-factor is much smaller.

In the RS model we have chosen the mass of the first $\mathrm{KK}$ mode $M_{1}=1.5 \mathrm{TeV}$ and the coupling $c_{0}=0.01$. In Fig. 4a we have plotted invariant mass distribution of the dilepton in the $\mathrm{RS}$ model. At the $\mathrm{KK}$ mode resonances the cross section differs from 
the SM cross section, but the dependence on the PDFs are very mild. In Fig. 4b the corresponding K-factors are plotted for various PDFs. The behaviour of the K-factor of the RS model can be understood with the help of Eq. (6 7). It is only in the RS graviton resonances region that $K^{(0)}$ is large and hence the $K$-factor is dominated by the $K^{G R}$ factor. In the off resonance regions it is the $K^{S M}$ which contributes. There is a wide difference in the $\mathrm{K}$-factor more in the second peak and even off peak where the effect is mainly SM. This is due to the high $Q$ value that is chosen in the RS case.

For the double differential with respect to rapidity and invariant mass, in Fig. 5 a we have plotted it for the rapidity range of $\mathrm{LHC}$ for $Q=1.5 \mathrm{TeV}$, which is the region of the first RS KK mode. It is only in the resonance region that the effects of RS are visible. Here there seems to be a clustering of PDFs but for CETQ in the central rapidity region. In the central rapidity region the K-factor varies from 1.6 - 1.75 Fig. 5 b. In the first RS KK resonance region at $Q=1.5 \mathrm{TeV}$ the gravity dominates and hence the $\mathrm{K}$-factor is large (Eq. (6) $)$ ). Beyond the central rapidity region $Y=0$ the $\mathrm{K}$-factor dependence on PDFs is substantial. In Fig. 5. we have plotted the double differential with respect to $\cos \theta^{*}$ for $Q$ fixed at the first resonance. The cross section is largest for $\cos \theta^{*}=0$ and MRST is the largest among the PDFs. The K-factor in Fig. $5 \mathrm{~d}$ is about 1.65 for wide range of $\cos \theta^{*}$ for Alekhin and MRST but for CTEQ it varies between $1.7-1.8$.

In the above we discussed the extra dimension effects at LHC, now we look at the Tevatron. For the ADD case, in Fig. [6 we have plotted the invariant mass distribution for the various PDFs. The spread due to the various PDFs over the $Q^{2}$ range is not too large. Only at large $Q$ there is some deviation from the SM result which is plotted in Fig. 6a. The K-factor for the $Q$ distribution for the various PDFs are plotted in Fig. [6], which are in tune with the SM K-factor at the Tevatron. In Fig. 6r we have plotted the PDF comparison plot for the rapidity distribution at $Q=0.7 \mathrm{TeV}$. CTEQ and MRST plots are very similar while Alekhin is larger in the central rapidity region. 
In the $Y=0$ region, the K-factor for CTEQ is about 1.1 while for MRST and Alekhin it is about 1.2, which is in the range of the SM K-factor, Fig. 6 $\mathrm{d}$.

For the RS model the PDF comparison plots are given in Fig. [7. In Fig. [7 w we have the invariant mass distribution and the deviation from the SM is only in the resonance region. The PDF dependence is very mild. In the first resonance region the K-factor (Fig. 7b) is dominated by $K^{G R}$ at $Q=0.7 \mathrm{TeV}$ but at Tevatron this value is not too different from the SM K-factor. In Fig. Tf the $\cos \theta^{*}$ distribution at the first resonance region is plotted, CTEQ and MRST overlap while Alekhin is larger over a wide range of $\cos \theta^{*}$. The K-factor Fig. [7d is in the range of the SM K-factor.

\subsection{Renormalisation/Factorisation scale uncertainties}

In Fig. 8a we have plotted the double differential $d^{2} \sigma / d Q d Y$ in the $Y$ range for LHC energies for a fixed $Q=0.7 \mathrm{TeV}$. The dependence of cross section on $\mu_{R}$ comes from the strong coupling constant at NLO and so at LO there is no $\mu_{R}$ dependence. At NLO $\mu_{R}$

dependence for the $Y$ distribution is plotted for the $\mu_{R}$ range $0.5 Q \leq \mu_{R} \leq 1.5 Q$. The $\mu_{R}$ spread is largest in the central rapidity region and would only reduce at the NNLO order level when the $\mu_{R}$ dependences would be compensated for by the dependence coming from the coefficient functions. In Fig. 8b we have plotted the K-factor for SM and $\mathrm{SM}+\mathrm{GR}$ and see how it dependence on $\mu_{R}$. The uncertainties due to $\mu_{R}$ is much larger when the gravity is included. The percentage spread is of the order of $3.5 \%$ which is comparable to the $\mu_{F}$ spread at NLO.

In Fig. 9] we have plotted $Y$ distribution and its K-factor for ADD and RS model at a fixed $Q=\mu_{R}$. The $\mu_{F}$ variation is studied by varying $\mu_{F}$ in the range $0.5 Q \leq \mu_{F} \leq$ 1.5 Q. We see that for both the ADD and RS model in going from LO to NLO in QCD, the uncertainties due to $\mu_{F}$ variation considerably get reduced. The spread of K-factor with $\mu_{F}$ is much smaller for the SM as compared to SM+GR. This certainly indicates need to go beyond NLO. Similar trends are observed for the $\cos \theta^{*}$ distribution plotted 


\begin{tabular}{|c|c|c|c||c|c|}
\hline \multirow{3}{*}{ ADD } & Distributions & \multicolumn{2}{|c||}{ Tevatron } & \multicolumn{2}{c|}{ LHC } \\
\cline { 3 - 6 } & & LO & NLO & LO & NLO \\
\hline \hline \multirow{3}{*}{ RS } & $d^{2} \sigma / d Q d Y$ & 22.8 & 7.4 & 9.5 & 3.5 \\
& $d^{2} \sigma / d Q d \cos \theta$ & 24.2 & 8.2 & 10.9 & 3.8 \\
\hline \multirow{2}{*}{${ }^{2} \sigma / d Q d Y$} & 23.2 & 7.7 & 18.7 & 6.9 \\
& $d^{2} \sigma / d Q d \cos \theta$ & 24.2 & 8.0 & 18.4 & 6.8 \\
\hline
\end{tabular}

Table 2: Percentage spread as a result of factorisation scale variation in the range $0.5 Q \leq \mu_{F} \leq 1.5 Q$. For the ADD case $Q=0.7 \mathrm{TeV}$. For the RS first resonance region $Q=1.5 \mathrm{TeV}$ for LHC and $Q=0.7 \mathrm{TeV}$ for Tevatron.

in Fig. 10,

In Table 2 we tabulate the percentage spread of the factorisation scale $\mu_{F}$ dependence in the range $0.5 Q \leq \mu_{F} \leq 1.5 Q$ for the LHC and Tevatron. On the average at LHC and Tevatron, the percentage spread of the scale variation get reduced by about 2.75 times in going from LO to NLO.

\section{Experimental Uncertainties}

In addition to the theoretical uncertainties that we have described in the previous section, there are uncertainties due to errors on the data. Various groups have studied the experimental errors and have estimates of the uncertainties on the PDFs within NLO QCD framework [10, 11]. Now that NLO QCD results are also available for extra dimension searches [4] for the dilepton production, we consider some of the distributions and estimate the uncertainties due to the experimental error. In Fig. 11] we have plotted the error band for the MRST 2001 PDF [11] in the ADD model for the dilepton invariant mass distribution at LHC. This error band is comparable to the 
spread associated with the different set of PDFs as given in Fig. 2a. At $Q=1 \mathrm{TeV}$ the percentage of experimental error is $7.5 \%$ for $S M+G R$ while the pure SM error is about $3.3 \%$. For the RS case at LHC in the first resonance region at $Q=1.5 \mathrm{TeV}$ the experimental error is about $12.8 \%$. At Tevatron the ADD model experimental error is $7.4 \%$ at $Q=1 \mathrm{TeV}$. The experimental error for this distribution for the central rapidity region is about $3.5 \%$ and is indicated in the Fig. 11b. In general the experimental error increases with the increase in $Q$.

\section{Conclusions}

We have studied the impact of various parton density sets at next to leading order in strong coupling constant $\alpha_{s}$ in QCD to one of the most important processes, namely Drell-Yan production of dileptons in hadron colliders such as LHC and Tevatron. This process can probe the physics beyond SM through exchange of new particles that these theories predict. At hadron colliders, the precise measurement of DY production cross sections is possible. In this context, we have studied the theories of extra dimensions such as ADD and RS which attempt to explain gauge hierarchy problem in SM. We have discussed various theoretical uncertainties that enter through renormalisation, factorisation scales and the parton density sets. We have quantified the uncertainties coming from various parton density sets using the recent results on NLO QCD corrections to parton level cross sections and recent PDF sets that take into account various theoretical and experimental errors. Our entire analysis is model independent thanks to the factorisation of QCD radiative corrections from the model dependent contributions. More precisely, our findings are independent of the finer details of the model as

they factor out from the rest. We find that the K-factor for various observable depends on the choice of PDFs.

\section{Acknowledgment:}

M C Kumar, CSIR JRF would like to thank CSIR, New Delhi for financial support. 


\section{References}

[1] N. Arkani-Hamed, S. Dimopoulos and G. Dvali, Phys. Lett. B249 (1998) 263;

I. Antoniadis, N. Arkani-Hamed, S. Dimopoulos and G. Dvali, Phys. Lett. B436 (1998) 257.

[2] L. Randall and R. Sundrum, Phys. Rev. Lett. 83 (1999) 3370; W.D. Goldberger and M.B. Wise, Phys. Rev. Lett. 83 (1999) 4922.

[3] Prakash Mathews, V. Ravindran and K. Sridhar, JHEP 0408 (2004) 048.

[4] Prakash Mathews, V. Ravindran, K. Sridhar and W.L. van Neerven Nucl. Phys. B713 (2005) 333.

[5] Prakash Mathews, V. Ravindran and K. Sridhar, JHEP 0510 (2005) 031.

[6] Prakash Mathews, V. Ravindran, hep-ph/0507250, to appear in Nucl. Phys. B.

[7] S. Alekhin Phys.Rev. D68 (2003) 014002.

[8] J. Pumplin et. al., JHEP 0207 (2002) 012.

[9] A.D. Martin et. al., Eur.Phys.J. C23 (2002) 73.

[10] M. Bothe, Eur. Phys. J. C14 (2000) 285; W. T. Giele, S. Keller, Phys. Rev. D58 (1998) 094023; W. T. Giele, S. Keller, D. A. Kosower, hep-ph/0104052; S. I. Alekhin, Phys. Rev. D 63 (2001) 094022; CTEQ Collaboration: J. Pumplin et al., JHEP 0207 (2002) 012; A. M. Cooper-Sarkar, J. Phys. G 28 (2002) 2669.

[11] A.D. Martin et. al., Eur.Phys.J. C28 (2003) 455.

[12] G. F. Giudice, R. Rattazzi and J. D. Wells, Nucl. Phys. B544 (1999) 3.

[13] T. Han, J. D. Lykken and R-J. Zhang, Phys. Rev. D59 (1999) 105006. 
[14] G. F. Giudice, A. Strumia, Nucl. Phys. B663 (2003) 377; G. F. Giudice, T. Plehn, A. Strumia, Nucl. Phys. B706 (2005) 455.

[15] V. M. Abazov et. al., DØ Collaboration, Phy. Rev. Let. 95 (2005) 161602.

[16] K. Cheung and G. Landsberg, Phys. Rev. D62 (2000) 076003.

[17] V. M. Abazov et. al., DØ Collaboration, Phy. Rev. Let. 95 (2005) 091801. 

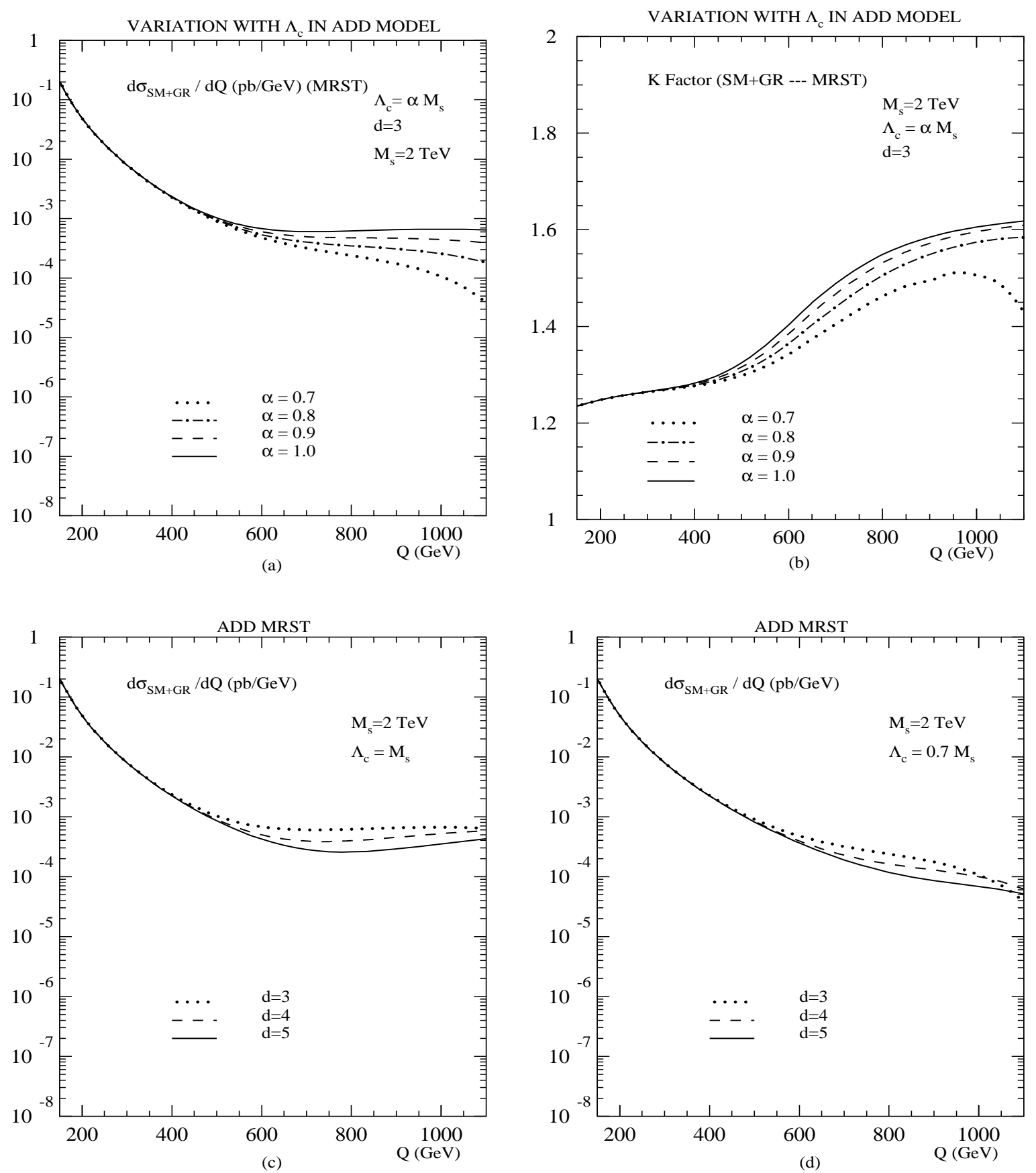

Figure 1: (a) Invariant mass distribution is plotted for various values of the cutoff $\Lambda_{c}=\alpha M_{s}$ in the ADD model. (b) The corresponding K-factor. (c) Invariant mass distribution as a function of the number of extra spacial dimension $d$ for $\Lambda_{c}=M_{S} \mathrm{TeV}$ at LHC. (d) The same plot as (c) for $\Lambda_{c}=0.7 M_{S}$. 

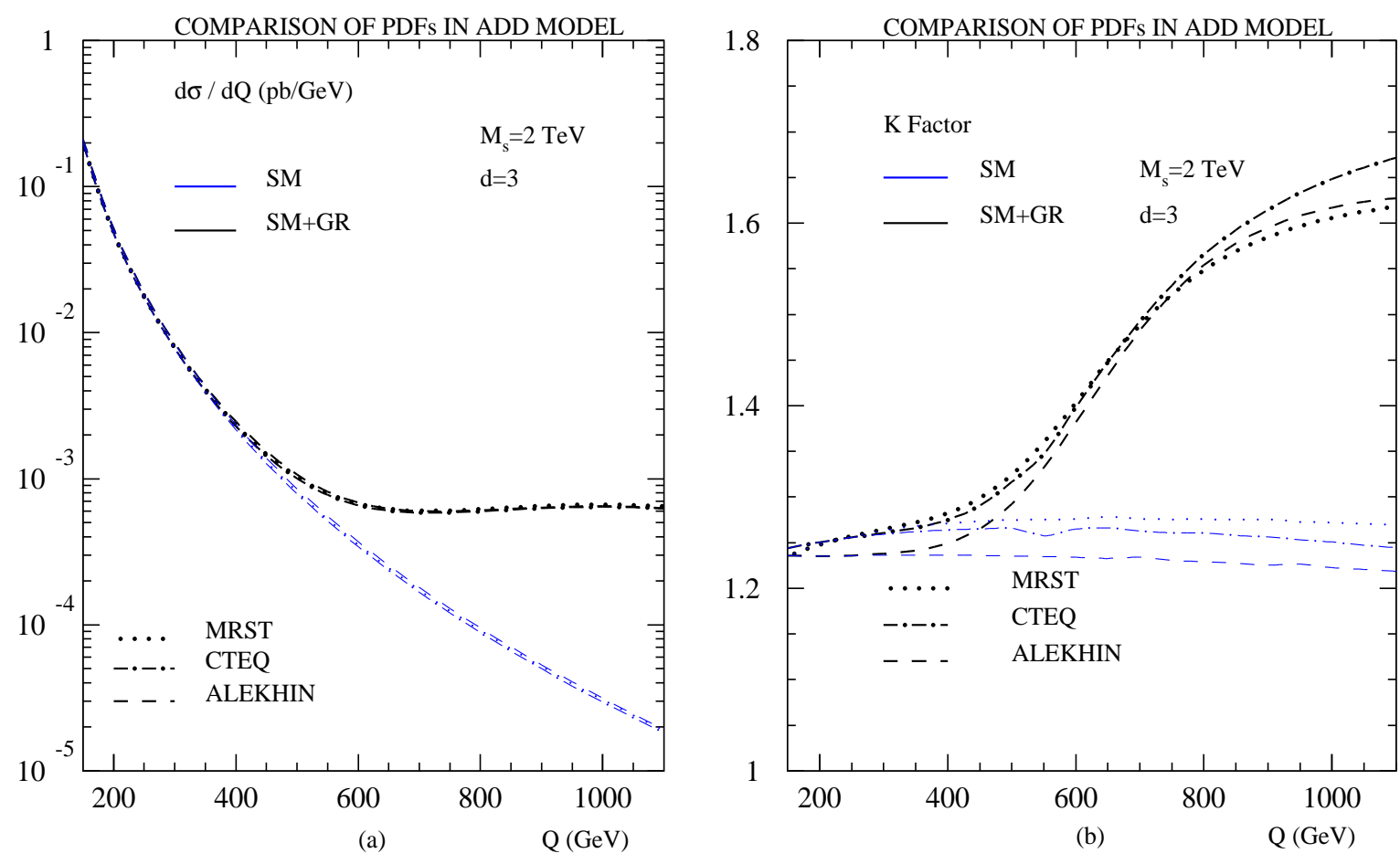

Figure 2: (a) Invariant mass distribution of the dilepton pair for ADD model with different PDFs to NLO in QCD. (b) The corresponding K-factor for the various PDFs. For both (a) and (b) we have plotted the SM background to NLO using the same line type in colour for the different PDFs. 

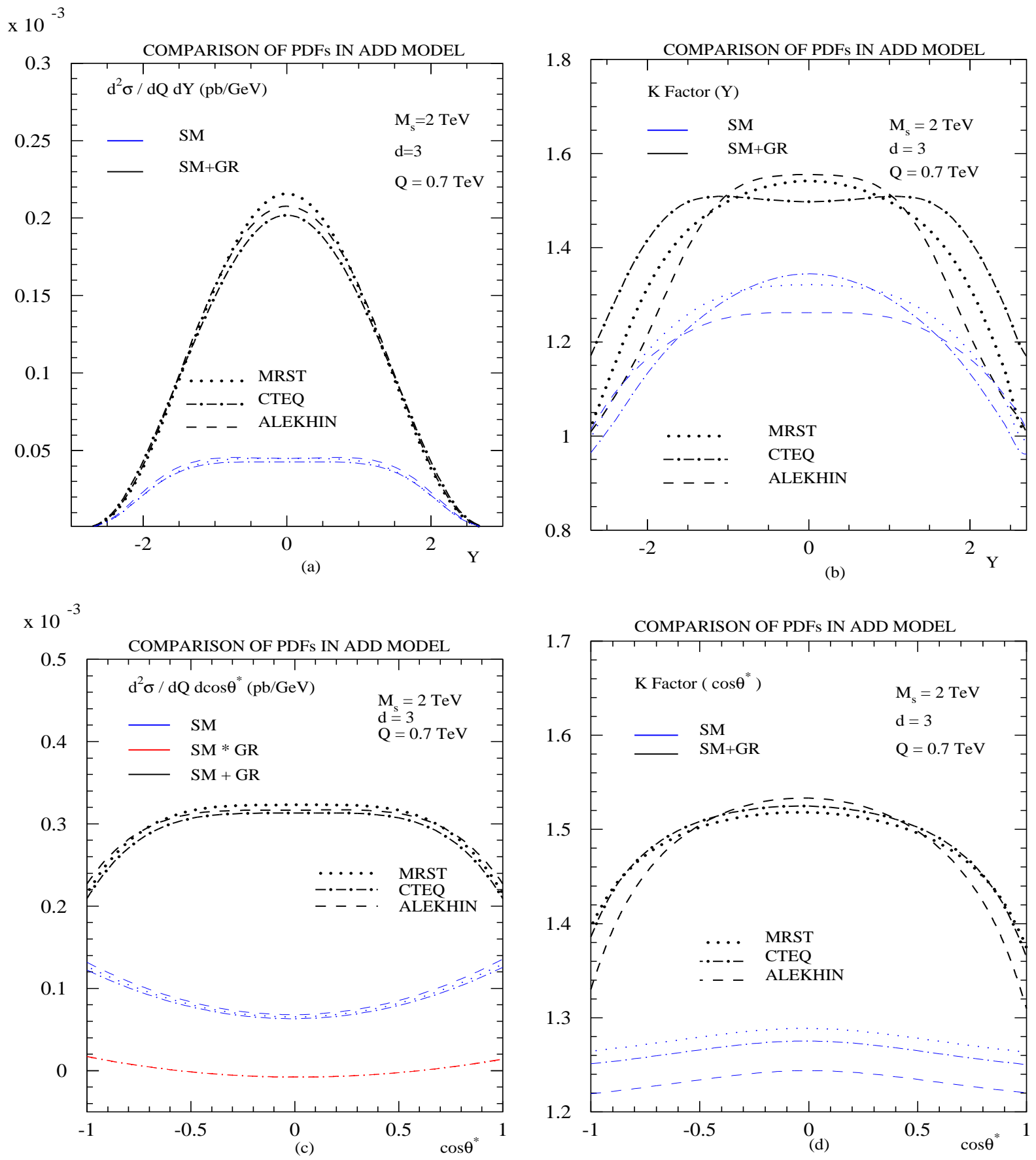

Figure 3: The comparison plots for the various PDFs sets for $Q=0.7 \mathrm{TeV}$ at LHC. (a) The double differential cross section with respect to invariant mass and rapidity as a function of rapidity. (b) The corresponding K-factor as a function of rapidity. (c) The angular distribution of the double differential cross section with respect to invariant mass and $\cos \theta^{*}$. The interference of the SM background and gravity effects is also plotted. (d) The corresponding K-factor. 

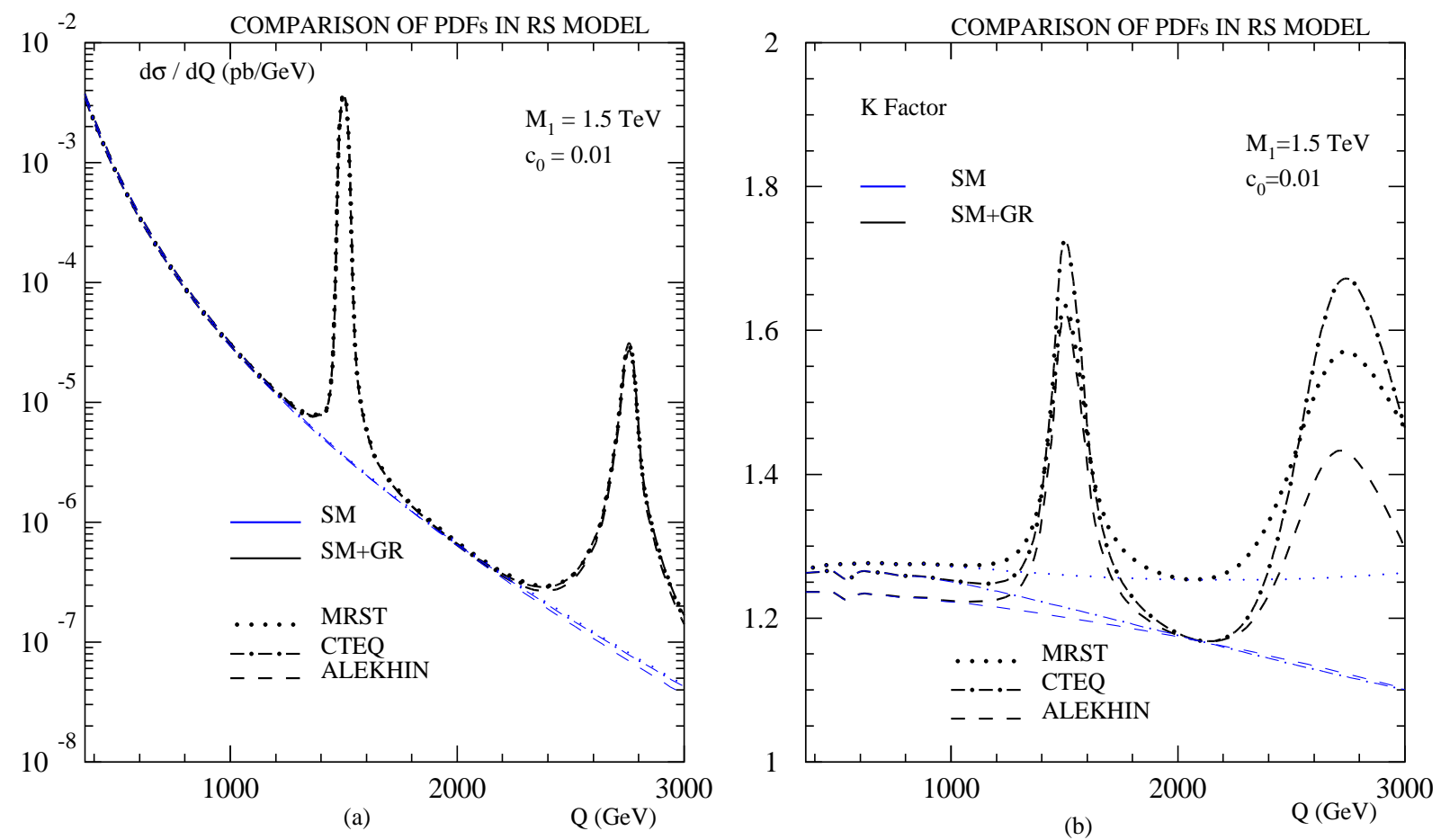

Figure 4: (a) The invariant mass distribution of dilepton pair production at LHC in the RS model for various PDfs. (b) The corresponding K-factor for the various PDFs. 

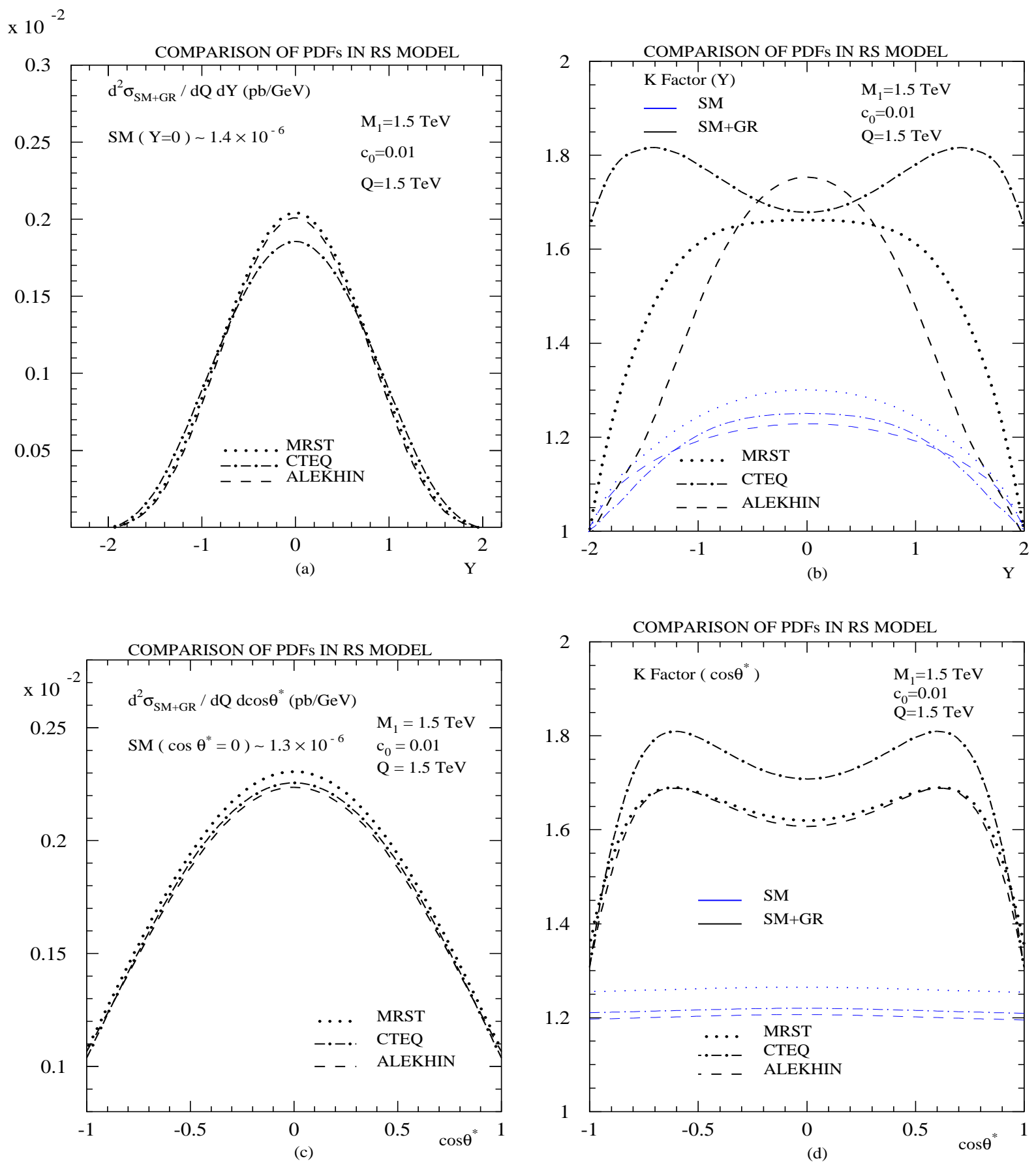

Figure 5: (a) The double differential cross section with respect invariant mass and rapidity for various PDFs in the $\mathrm{RS}$ model at $Q=1.5 \mathrm{TeV}$, the region of first resonance. (b) The corresponding K-factor as function of rapidity at $Q=1.5 \mathrm{TeV}$. (c) In the region of first RS resonance, the double differential with respect to invariant mass and angular distribution of the lepton is plotted for the various PDFs at LHC. (d) The corresponding K-factor for the various PDFs. 

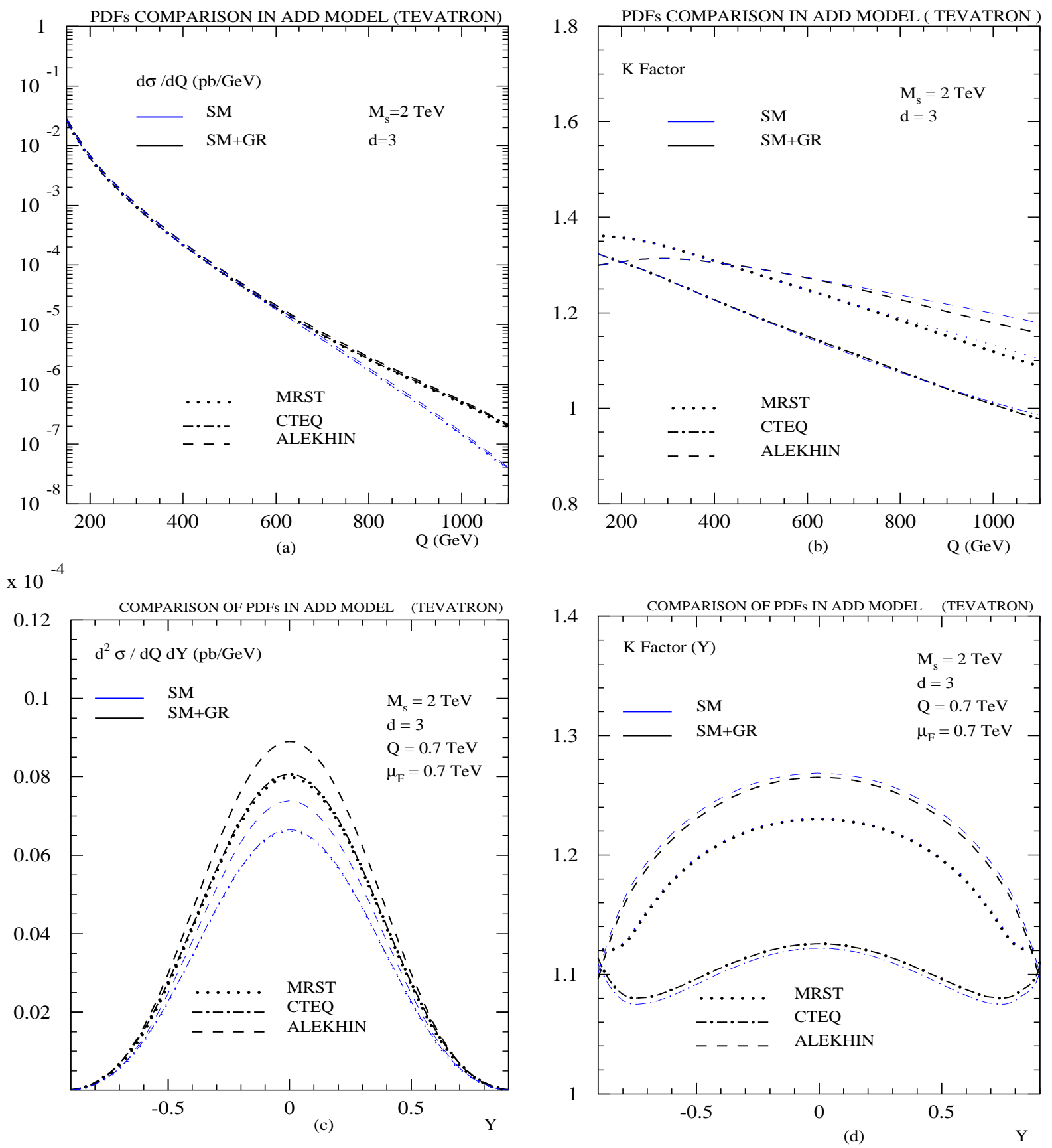

Figure 6: ADD model at Tevatron for the various PDF set, we plot in (a) the invariant mass distribution. In (b) the corresponding K-factor. (c) The double differential with respect to $Q$ and $Y$ is plotted for a fixed $Q=0.7 \mathrm{TeV}$ and for the $Y$ range of Tevatron. In (d) the corresponding $\mathrm{K}$ factor is plotted. 

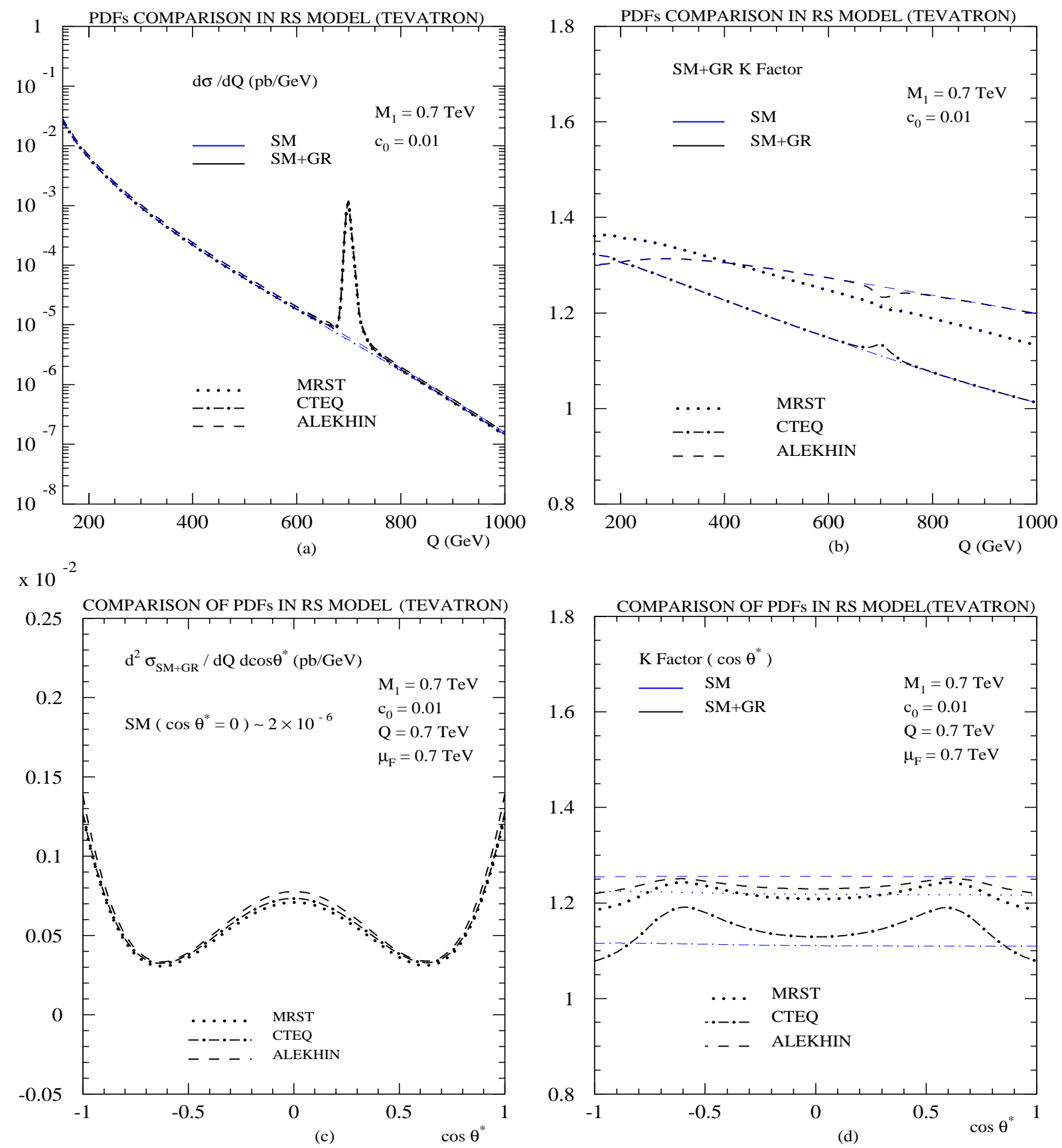

Figure 7: RS model at Tevatron for the various PDF set, we plot in (a) the invariant mass distribution. In (b) the corresponding K-factor. (c) The double differential with respect to $Q$ and $\cos \theta^{*}$ is plotted for a fixed $Q=0.7 \mathrm{TeV}$ and for the $\cos \theta^{*}$. In (d) the corresponding $\mathrm{K}$ factor is plotted. 

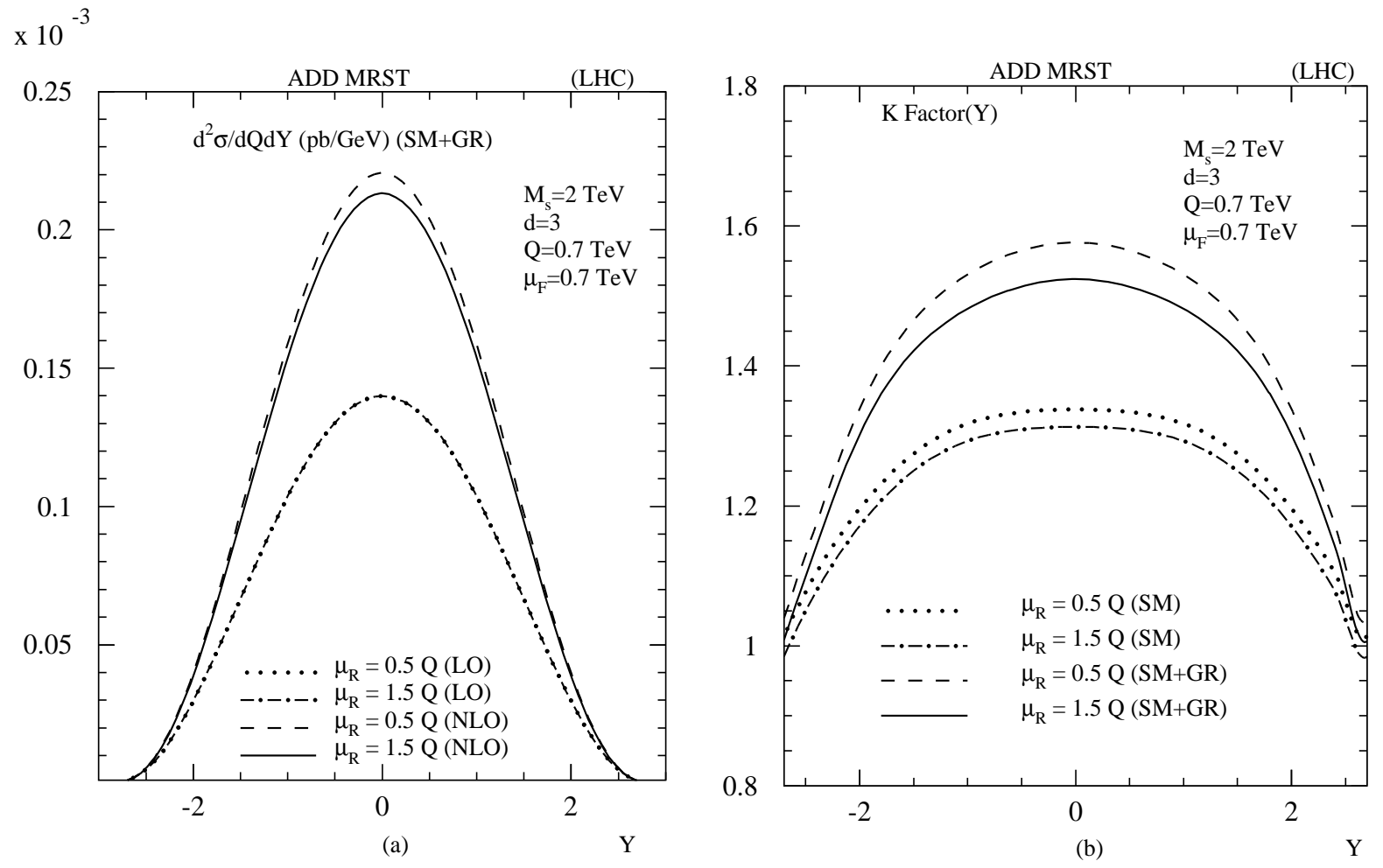

Figure 8: The renormalisation scale dependence for double differential cross section as a function of rapidity for $Q=0.7 \mathrm{TeV}$. The PDF used is MRST and the renormalisation scale is varied in the range $\mu_{R}=0.5 Q-1.5 Q$ for LO and NLO. (b) The K-factor dependence on $\mu_{R}$ for both SM and $\mathrm{SM}+\mathrm{GR}$. 

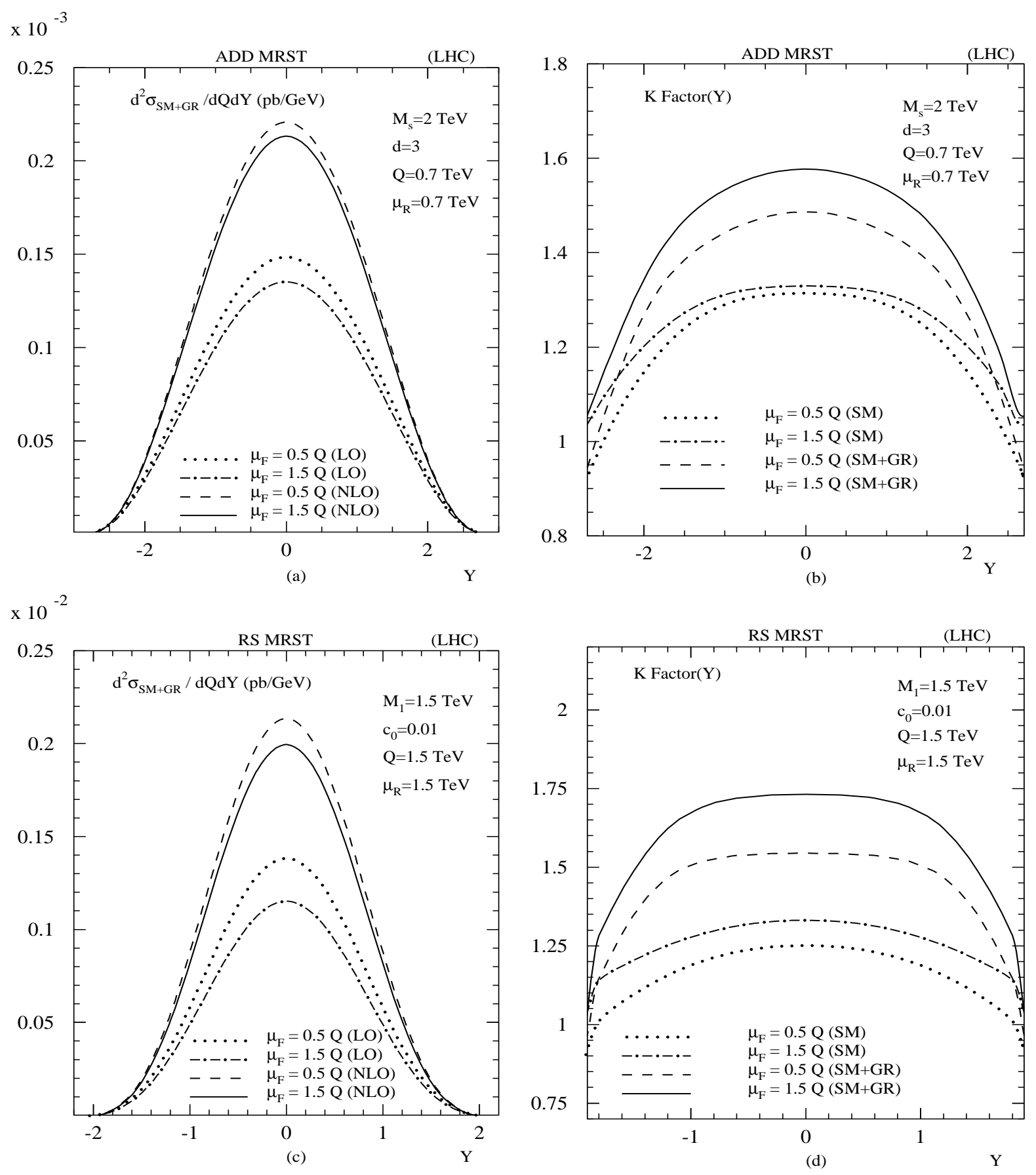

Figure 9: (a) Factorisation scale dependence for the double differential cross section as a function of rapidity for LO and NLO for factorisation scale in the range $\mu_{F}=$ $0.5 Q-1.5 Q$. (b) SM and SM+GR K factor for ADD rapidity distribution in the same variation of $\mu_{F}$. In (c) the RS distribution at $Q=\mu_{R}=1.5 \mathrm{TeV}$ in the region of first resonance. (d) The SM and SM+GR K factor for RS rapidity distribution. 

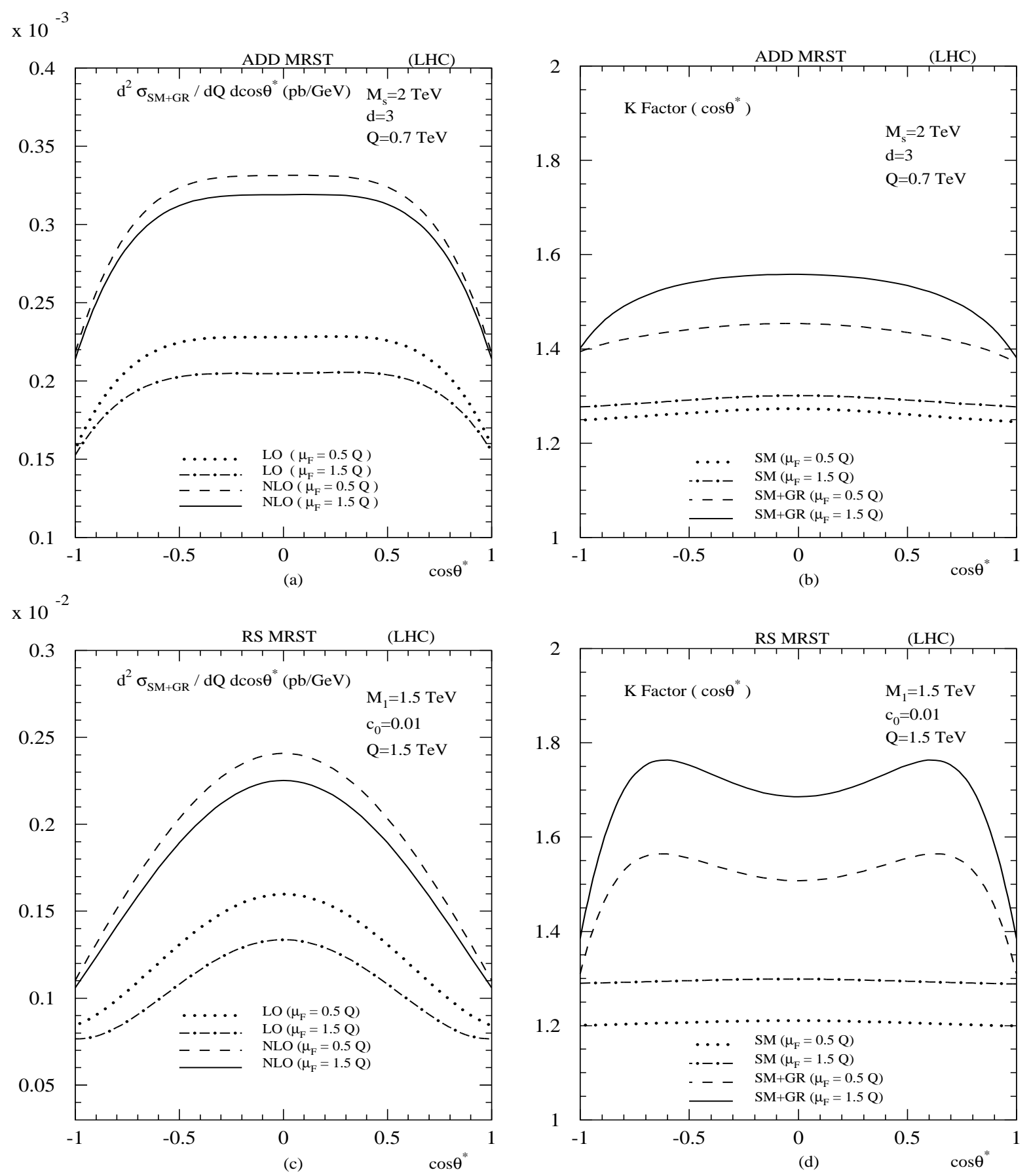

Figure 10: (a) Factorisation scale dependence for the double differential cross section as a function of $\cos \theta^{*}$ for $\mathrm{LO}$ and NLO for factorisation scale in the range $\mu_{F}=$ $0.5 Q-1.5 Q$. In (b) we have plotted the SM and SM+GR K factor for ADD at $Q=\mu_{R}=0.7 \mathrm{TeV}$. In (c) the RS $\cos \theta^{*}$ distribution for LO and NLO in the same range of $\mu_{F}$. (d) The $\mathrm{SM}$ and $\mathrm{SM}+\mathrm{GR}$ K-factor at $Q=\mu_{R}=1.5 \mathrm{TeV}$, the region of first resonance. 

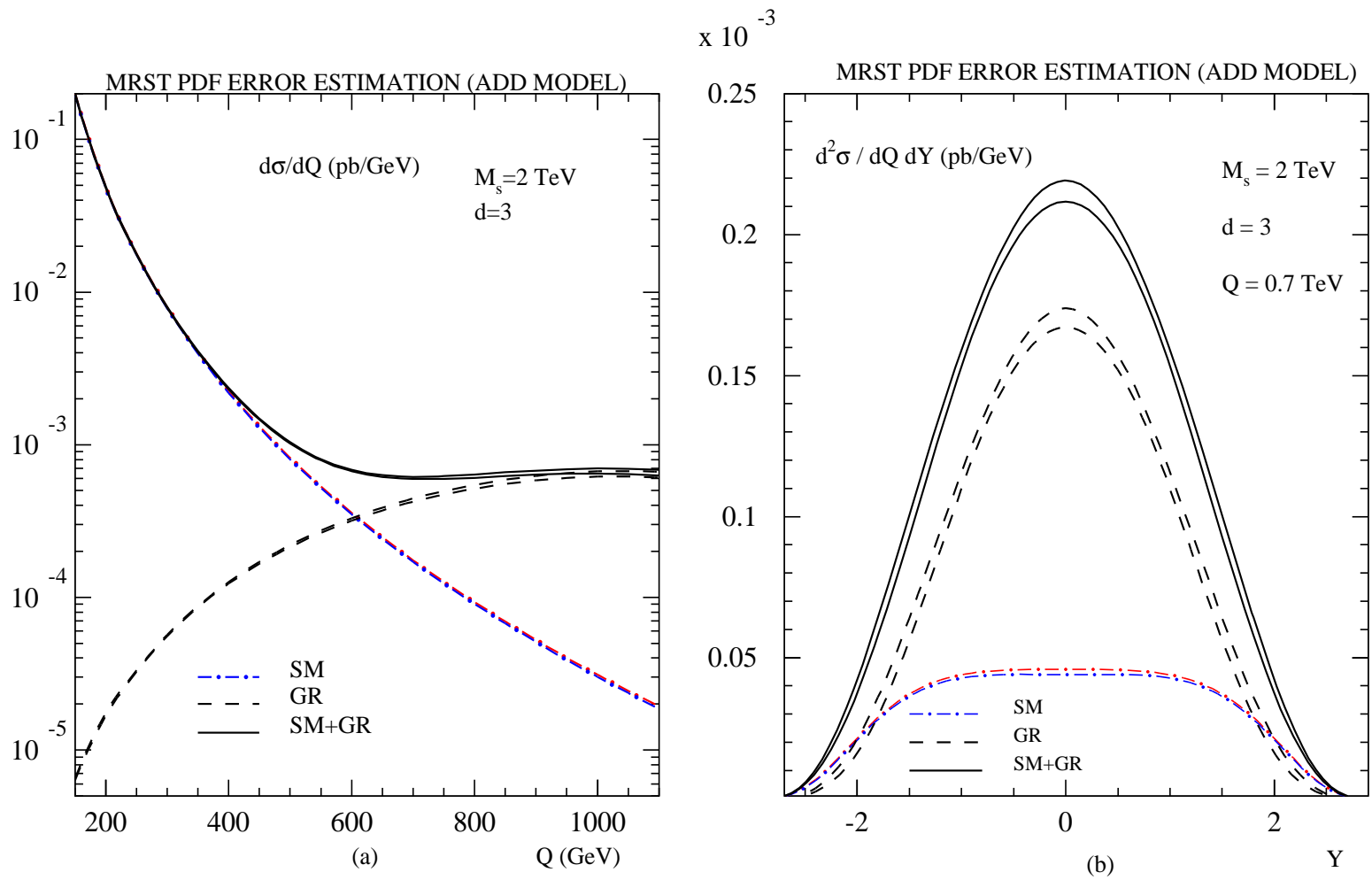

Figure 11: The experimental error on the MRST PDF at LHC in the ADD model for (a) The invariant mass distribution and (b) The rapidity distribution for a fixed $Q=0.7 \mathrm{TeV}$. 